\title{
The Foundations of Concordance Views of Phylogeny
}

\author{
Joel D. Velasco*
}

Despite the enormous importance and widespread use of the term, it is unclear exactly what a phylogeny is and what a phylogenetic tree represents. A natural thought is that a phylogeny should represent the "dominant history" of the flow of genetic information. Here, I will focus on methods in which a tree is determined by input gene trees. I compare various possibilities of how to do this including consensus trees, the $R^{*}$ triplet method, and concordance methods. I make precise the notion of a concordance factor and then compare concordance trees to species trees, which attempt to track population histories through time. I show that even in the most idealized circumstances, it is possible for the primary concordance tree to differ from the species tree. I argue that when these trees differ, there are reasons to think of the primary concordance tree as a better representation of phylogenetic history than the species tree and that when these highly idealized conditions break down, it is especially plausible that we should take concordance trees seriously as an interpretation of phylogeny and as the basis for taxonomic grouping.

\section{Keywords}

phylogeny $\bullet$ concordance tree $\bullet$ clade $\bullet$ plurality consensus

Part of the special issue Species in the Age of Discordance, guest-edited by Matthew H. Haber and Daniel J. Molter.

Despite the enormous importance and widespread use of the term, it is unclear exactly what a phylogeny represents. It is important to define phylogeny precisely since other central terms like "clade" and "monophyletic" are often defined relative to phylogenetic trees and on some views in taxonomy, taxa (or at least taxa above the species level) must be clades. Edwards (2009) presents the common picture in contemporary systematics as depending on the existence of a "species tree" in which phylogeny "records the branching pattern of evolving lineages through time" (2). But what, precisely, does this mean?

*Department of Philosophy, Texas Tech University, 2500 Broadway, Lubbock, Texas 79409, USA, joel@joelvelasco.net

Received 21 November 2017; Revised 25 November 2018; Accepted 8 March 2019 doi:10.3998/ptpbio.16039257.0011.020 
A phylogenetic tree depicts genealogical relationships between taxa. Such a tree is meant to be a useful simplification of the vast network of life - the history of the genealogical connections between all organisms. Obviously this network is not itself a phylogenetic tree-nodes in a tree have at most one parent while many organisms have two parents-but a natural thought is that we could construct the tree from this network. This is implicit in, for example, the diagram from Hennig (1966) depicting a lineage split and in all of the "species tree" literature where we attempt to infer the species tree from genetic data using coalescent theory.

But it is unclear exactly how this connection works. For example, we could try to first define species and then define what branching is in terms of speciation. But some authors explicitly say that this conception of a species tree is independent of the debates about species (Edwards 2009). The problem is due to the fact that this would make phylogeny dependent upon classification when they should be independent-or indeed, the dependence might even go in the other direction (Velasco 2008).

The more natural direction would be to directly define lineages in terms of organismal genealogy and then define lineage splitting. Then after we understand the branching pattern, we could identify species from this phylogeny if we wish, though we wouldn't have to. A species concept defined in this way where a species is a lineage segment between two branching events (or nodes on the tree) is called an "internodal species concept" (Ridley 1989 calls his own view along these lines the "cladistic species concept"). Mathematically, starting from a full organismal graph with a set of parent-offspring relations, it is always possible to put the organisms into non-overlapping clusters satisfying various kinds of criteria that you might care about and then to take these clusters and put them into a hierarchy which is equivalent to a phylogenetic tree (see e.g., Bui-Xuan et al. 2012). Kornet (1993) gives one way to do this that intuitively matches well with the internodal concept, and which Kornet et al. (1995) claim uniquely identifies the internodal concept. Steel (2007) and Dress et al. (2010) look at the problem from a more abstract graph-theoretic perspective, and show that various constructions of trees from organismal networks are always possible. Ignoring the problem of what constitutes a species, we could directly define a hierarchy and therefore clades and taxa from this graph.

Alternatively, Baum $(2009,78)$ and Velasco (2009) explore a different way of starting with some set of contemporary organisms and constructing exclusive groups of these organisms in order to build synchronic clades. In this approach, organisms in clades will always end up more closely related to other organisms in the same clade than to any outside the clade. This seems a desirable consequence but need not be true in the internodal style treatments. On this picture, the exclusive groups still form clusters and these clusters can be put into a tree/hierarchy. But this will not track lineages through time in the way that internodal views do.

Both types of views mentioned above can lead to a precise definition of a phylogenetic tree that is constructed from the genealogical history of organisms. But a natural thought is that a phylogeny should represent the flow of genetic information and that this is, in some sense, more fundamentally what evolutionary history is about. As is well known, if we define phylogeny in terms of the genealogical histories of organisms, then gene tree topologies could differ from the species tree topology by a number of different processes, for example, incomplete lineage sorting and lateral gene transfer. Some of these causes of discordance are nicely summarized in Maddison (1997). After examining various kinds of genetic discordance, Maddison suggests that this might cause us to re-examine what we really mean by "phylogeny". Organismal genealogies tell us what sorts of genetic histories we might expect to see. But isn't the actual flow of genetic information more important than the expected flow?

For example, one fundamental criticism of an organismal genealogy based approach is that in sexually reproducing populations, ancestry per se does not determine the genetic make-up of 
individuals - for instance, for any person, it is likely that at least one of their ancestors twelve generations in the past will have contributed nothing to their genome (Donnelly 1983).

With this kind of example in mind, Baum (2009) argues for the genetic approach as follows:

Although I can see arguments in both directions, I favor giving primacy to the gene genealogical criterion. Organismic ancestry constrains how genetic ancestry should be structured under Mendelian inheritance and in the absence of selection. When the realized patterns of genetic relatedness deviate from expectation, I think we should recognize taxa based on what actually happened rather than worry about what should have happened. In particular, it seems preferable to adopt gene genealogical exclusivity, which is sensitive to a history of selection, rather than organismic exclusivity, which is not. Additionally, gene genealogical exclusivity is more tractable in practice because we can relatively directly evaluate actual genomic ancestry, for example, using concordance analysis (Ané et al. 2007). Thus, I believe that gene genealogical exclusivity provides a more helpful and more tractable criterion for grouping organisms into species and other taxa. (5)

The difference between what is expected to happen and what actually did happen is even clearer when we examine lateral gene transfer or endosymbiosis, which are simply ignored in definitions of phylogeny based on organismal history. The basic philosophy underlying the idea that phylogenies ultimately just represent the history of genetic inheritance is discussed in papers such as Maddison $(1995,1997)$. As Maddison $(1995,285)$ puts it, "one possible interpretation of a species phylogeny is that it depicts the lines by which genetic information was passed on and nothing more". But it is unclear from these papers how to turn this philosophical idea into an explicit definition of phylogeny.

I will not engage here in a debate about whether defining phylogenies from gene histories is ultimately preferable to organismal histories. Some of the relevant issues will be discussed later in the paper. For now, it is enough to realize that they are different. With this in mind, I now turn to the project of how to define a phylogeny by looking at the actual flow of genetic information. The most promising approaches in the literature along these lines are the views developed by David Baum and others using concordance factors to which I now turn. First, I will carefully explicate the concept of a concordance factor and a concordance tree and finally, compare this approach to the species tree approach that we started with.

\section{Baum's "Concordance Tree" Idea}

Concordance views get their start from Baum and Shaw (1995) who sought to define species as genealogical units of phylogeny. The idea was that species lie at the boundary between divergent and reticulate genealogies. As Baum and Shaw argue, if we want species to be taxa, then they should be clades where any organism in the group can "represent" the group from the standpoint of systematics. This can happen only if all of the organisms in the group belong in the same place on a phylogenetic tree. The groups are thus "exclusive"-organisms in the group are more closely related to each other than to any outside the group. While de Queiroz and Donoghue (1990) defined exclusivity in terms of the recency of (organismal) common ancestry, Baum and Shaw define exclusivity as: "A group of organisms is exclusive if their genes coalesce more recently within the group than between any member of the group and any organisms outside the group" (296). 
If by "their genes" we mean all of their genes, then this definition is too strict to be a necessary condition for taxa (e.g., humans would not count as a species or any other taxon). Shaw (2001, 881) suggests that perhaps a group is exclusive "once the majority of gene copies each find their closest genealogical relative within that population" while Baum (2007) relaxes this definition further and says "I wish to propose that a primary concordance tree, a tree composed of clades that are true for a plurality of the genome, provides a useful first order summary of the primary history." (418). To describe this more carefully, Baum introduces the quantitative notion of a "concordance factor" - "the proportion of the genome for which a given clade is true." Since this is a proportion, concordance factors fall between 0 and 1 with a concordance factor of 1 corresponding to the "all of their genes" condition in Baum and Shaw (1995). By a "plurality", Baum means to say that the concordance factor of the clade is higher than the concordance factor of any contradictory grouping. Baum (2009) and Velasco (2010) are explicit that taxa are clades on the primary concordance tree defined using the plurality notion and thus can have concordance factors of less than 0.5 .

Philosophical arguments for the view that we should think of the phylogenetic tree as the primary concordance tree can be found in Baum (2009) and Velasco (2010). But it turns out their definitions of phylogeny are still not precise and are crucially ambiguous, so before we carefully explore the consequences of this view there are some definitional issues that must be settled first. Exploring the basic purpose behind developing a concordance view will interestingly lead us to multiple, distinct, possible definitions.

Recall that our original definition is that "A group of organisms is exclusive if their genes coalesce more recently within the group than between any member of the group and any organisms outside the group" (Baum and Shaw 1995, 296). What exactly do we mean by "their genes" in the definition of exclusivity? Do we mean every gene that is in every organism in the group? Or any gene that is in any organism? These can differ once we recognize the fact that one organism might have a gene that is not homologous with any gene from some other particular organism. Almost all of the work done explicitly on concordance factors assumes that each organism contains an identical set of genes. Ané et al. (2007) and other papers that use concordance analysis with empirical data recognize that this is simply false in real cases, but they treat this as a case of "missing data" which does not fundamentally change the meaning of what a concordance factor is. To better understand the work that has already been done, for now, let us assume that each organism in question has exactly the same set of genes. This puts us in the standard "consensus" framework in which we have a set of input trees with the same leaf set and we want to construct a new consensus tree. There are a number of ways one might form a tree from gene tree inputs. For example, we could simply take the most frequent gene tree-or even a random tree. Here, I examine only methods that are based on shared clades on these gene trees and not necessarily the whole gene tree itself.

When each gene tree has an identical leaf set, the natural definition of a concordance factor is the following: For any set of organisms $A$, we say that $c f(A)$ (the concordance factor) is the proportion of loci for which $A$ is a clade in the tree for that locus. We also say that two sets overlap if and only if they intersect, and neither set is contained in the other (formally, $A$ and $B$ overlap if none of the three sets $A \cap B, A-B$, and $B-A$ is empty). Two sets that overlap are also sometimes referred to as incompatible since no rooted phylogenetic tree can contain both of them simultaneously as clusters.

Now we can define five concordance (consensus) methods. Here the term 'consensus' method signifies that we are assuming that each input gene tree has the same leaf set:

- Strict consensus: A strict cluster is any set $A$ of organisms such that $c f(A)=1.0$. The strict consensus tree is the hierarchy formed from all and only the strict clusters. 
- Majority consensus: A majority cluster is any set $A$ of organisms such that $c f(A)>$ 0.5. The majority consensus tree is the hierarchy formed from all and only the majority clusters.

- Majority(+) consensus: A majority (+) cluster is any set $A$ of organisms such that $c f(A)>$ $c f o(A)$, where we let $c f o(A)$ denote the proportion of loci for which $A$ overlaps some cluster of the tree at that loci.

- Plurality consensus: A plurality cluster is any set $A$ of organisms such that $c f(A)>c f(B)$ for any $B$ which overlaps with $A$. The plurality consensus tree is the hierarchy formed from all and only the plurality clusters.

- Greedy consensus: A greedy consensus tree is any tree obtained from the clusters present in trees across the loci, by ranking them according to their concordance factor, and constructing a set of clusters, beginning with the cluster of maximal $c f$-value, and adding further clusters in the order of their diminishing $c f$-values, omitting any clusters that overlap with any of the clusters so far accepted. Ties are broken arbitrarily.

The literature on consensus methods is vast, with Bryant (2003) providing a helpful survey. Each of these methods is always guaranteed to yield a tree (though the greedy consensus method will not always yield a unique one). Dong et al. (2010) investigated the relationship between the plurality consensus method (there called the "frequency difference" method) and the other methods. The conclusion is that as indicated by its placement in the above list, the plurality consensus tree is always a refinement (and possibly a proper one) of the Majority (+) tree and in turn it is refined by any greedy consensus tree (again, this is possibly a proper refinement). Recall that one tree is said to refine another if all of the clusters in the latter tree are clusters of the former.

While these methods will always yield a tree in the consensus setting when each taxon has the same set of genes, this is no longer true in the more general setting where some input trees might contain different genes. Methods that work under these more general conditions are known as "supertree methods" and a number of such methods are well-studied. For a survey, see Bininda-Emonds (2004). It is interesting to point out that under a natural definition of concordance factor in the supertree setting, the plurality consensus method (unlike the majority or strict consensus methods) has a natural generalization which is still guaranteed to form a tree (Steel and Velasco 2014).

As was pointed out earlier, we want to weaken our definition of a clade to include more than just strict clusters. This allows some gene trees to conflict with the phylogenetic tree. But how much conflict should be allowed before we say it is too much and it destroys the integrity of a clade? Here we will focus on the suggestion of Baum (2007) to use the plurality consensus tree, which allows that a group might be a clade even though it has a concordance factor of cf $<0.5$. There are good reasons to allow seemingly low concordance factors for groups that we might consider to be clades. Imagine a well-mixed population of, say, 1,000 organisms that splits into two- perhaps because of a new geographical barrier arising or because of a migration event. Initially, it is likely that neither population will have concordance factors of any significance. Decent sized populations may even have concordance factors of zero and perhaps no group at all even has $c f>0.01$ (besides small family groups). But once there is a reproductive barrier in place, the concordance factors of isolated groups will gradually start to rise more or less continuously. Eventually they may reach the 0.5 threshold. But nothing of biological significance happens when the population has $c f=0.49$ and no other groups are above $c f=0.01$, 
and then it moves from $c f=0.49$ to $c f=0.50$. In many such cases, it makes sense to grant the status of clade to these groups even before they reach the 0.5 threshold. Indeed, it would not be surprising at all if many groups traditionally named as taxa and treated as clades exhibit concordance factors of less than 0.5 .

So we should think about ways that we might try to take account of such groups. A necessary condition is to loosen the requirement that the concordance factor must be greater than 0.5 . Perhaps the most obvious way to loosen the requirement turns out not to work. If we tried to actually set a lower threshold (say clusters are all groups with $c f$-score $>1 / 3$ ) then it would not be guaranteed that these clusters form a hierarchy. This does not mean merely that there could be an unresolved tree. Rather, setting the threshold below 0.5 allows for the possibility that the clusters cannot be identified with clades on a tree since they can overlap in the technical sense. But there are many ways to refine the majority tree. So here, we reach a philosophical decision about the best way to proceed.

One possible refinement is the plurality consensus tree as defined above. But there are other ways that we might try to allow for these clades. Velasco (2010), for example, argues that genetic exclusivity may be based on the genealogical relationships between the organisms in the group. He suggests that for $a$ and $b$ to be in the same taxon, they have to be more closely related to each other than to any organism outside the group. But this organismal triplet-based definition can have some strange consequences. For example, organisms could be closely related to different members of the group for different reasons. In the extreme case, a clade on the primary concordance tree might not be a clade for any gene tree at all (if, for example, $a$ and $b$ are close on genes $1-5, b$ and $c$ on genes $6-10$ and $a$ and $c$ on genes 11-15). While many discussions of the "genealogical species concept" describe the view as being defined by exclusivity, which is informally described in this triplet comparison between organisms, in practice, this turns into a definition based on the "reciprocal monophyly of gene trees" which is a concordance factor based definition. Many authors assume these are the same (for example, Hudson and Coyne 2002 and Velasco 2010), but as I will now show, they are not the same.

A natural way to formalize this triplet-based reading of exclusivity is by the $R^{*}$ consensus method discussed in several places including Bryant (2003) and Degnan et al. (2009). We define " $a$ is more closely related to $b$ than to $c$ " as saying that there are more rooted triples $a b \mid c$ than either $a c \mid b$ or $b c \mid a$. Then we form clusters where for every $a, a^{\prime}$ in $A$ and every $b$ not in $A, a$ is closer to $a^{\prime}$ than to $b$. Formally, let us say that $A$ is an $R^{*}$ cluster if for all $a, a^{\prime} \in A$ and all $b$ not in $A$, the proportion of $i$ for which $a a^{\prime} \mid i b$ is true is greater than the proportion of $i$ for which $a b \mid i a^{\prime}$ holds or the proportion of $i$ for which $a^{\prime} b \mid i a$ holds. It can be shown that the $R^{*}$ clusters form a unique hierarchy and thereby a tree. As an example to describe how the $R^{*}$ consensus method works, Degnan et al. (2009) take gene trees $(((a b) c) d),(((a d) c) b),(((b c) a) d)$, and $(((c d) a) b)$ as input. As they explain, the $R^{*}$ consensus tree is $((a c) b d)$ where $a c$ forms a clade despite not being a clade on any of the input gene trees. Since this means that $c f(a c)=0, a c$ is not a plurality cluster and so these two methods ( $R^{*}$ and plurality consensus) differ.

So now we have two distinct methods that allow for clades to have concordance factors of less than 0.5 . Both are refinements of the majority consensus tree, which means that any group with a concordance factor of $>0.5$ will automatically be a clade using either of these methods. Both of these methods also arose naturally from thinking about how to construct a phylogeny that represents the dominant gene history. So both are reasonable possibilities for definitions of clade or of exclusive group.

It is also interesting to compare these definitions to particular algorithms developed for concordance analysis in the literature. The first such algorithms were given in Ané et al. (2007). The authors of this piece (including David Baum) cite Baum (2007) as providing the inspiration for 
concordance analysis and they claim to be implementing a particular procedure for estimating "the primary concordance tree". Their program, BUCKy (updated in Larget et al. 2010), implements a particular "greedy consensus" algorithm in order to construct the concordance tree. They first calculate an estimated (posterior probability) concordance factor for each possible clade and then add these clades to a tree starting with those with the highest $c f$-scores and then continuing to add clades of lower estimated cf values as long as they are consistent with the tree (when there is a tie, the choice of which clade to add is arbitrary). This procedure produces a greedy consensus tree- - a fully resolved tree that is a refinement of the majority consensus tree.

While this procedure yields a tree of some interest for classification, it can fail to correspond to either the $R^{*}$ or plurality consensus methods above. In particular, it can differ from the plurality consensus tree by adding a clade when there is some conflicting clade that has a higher concordance factor. This can occur when that latter clade with a higher $c f$-value has already been rejected because it is incompatible with a third clade with a yet higher $c f$-value.

For a simple example, imagine four taxa (organisms) $a, b, c, d$ with the same set of genes where the percentage of gene trees work out to be the following:

$$
(a b)(c d)=48, a(d(b c))=49, c(d(a b))=3, \text { and all others }=0 .
$$

Now $c f(a b)=0.51$ and so it is a cluster. But any fully resolved tree that has $(a, b)$ as a clade will have at least one clade that conflicts with the clade $(b, c)$, which has a concordance factor of 0.49 . The plurality consensus tree is thus $(a b) c d$ with an unresolved trifurcation. But the greedy consensus method will see that $(c, d)$ has a $c f$-score of 0.48 , which is greater than the score of the conflicting possibilities $(a, b, c)$ and $(a, b, d)$, and so it will output $(a b)(c d)$ as the concordance tree despite the fact that $c f(c d)<c f(b c)$.

This may not seem so bad, but with enough taxa, we can get groups with arbitrarily low concordance factors to be added to the greedy consensus tree which conflict with concordance factors as high as 0.5 . We can see an additional problem with greedy consensus trees in this context when we recall what it is we are trying to do when we perform a concordance analysis. We are trying to determine which sets of organisms genuinely form clades. There is no reason that an arbitrary collection of organisms should necessarily form a fully resolved tree! What if the collection we started with was 100 randomly selected humans? We may find small clusters of them that turn out to be all closely related to each other, but we should certainly expect many groups to be such that no subgroups form clusters. Similarly, if we looked at ten humans, ten chimps, and ten gorillas, with the right organisms, it should be possible to detect three cladesone for each species-but no further clades within these groups. The purpose of concordance analysis is hidden when we do an analysis on a set where each of our samples comes from traditionally separate taxa. It may be that according to a concordance analysis, some of your samples really should not be in separate taxa. But a greedy consensus method forces us into a fully resolved tree.

Larget et al. (2010) and Ané (2010) recognize that there is a problem with greedy consensus, but the problem they discuss stems from the claim of Degnan et al. (2009) that greedy consensus is statistically inconsistent as an estimate of the species tree. So as of June 2010, BUCKy has been modified to implement an algorithm using unrooted quartets. This method is known as $Q^{*}$ (Bryant 2003) and is closely related to $R^{*}$. But as we have already seen, $R^{*}$ also differs from the plurality consensus tree, which is the more natural interpretation of the explications in Baum (2007) and Baum (2009).

There are, of course, other ways to refine the majority tree. But these three ways-plurality consensus, $R^{*}$ and greedy consensus-represent the most natural refinements based on some reasonable principles and they have each been discussed in the literature. If we use gene trees to 
identify clades for systematic purposes, it is clear that the greedy consensus method creates far too many clades. But $R^{*}$ and plurality consensus are both reasonable ways of defining exclusive groups which could serve as clades. Concordance factors are designed to make sure that there is some set of genes that unites everything in the clade. The $R^{*}$ tree doesn't guarantee that; instead it ensures that for any pair of organisms in a clade, there is a reason that they belong together-though the reasons may be different for different organisms. Rather than debate the merits of each method here, we instead move to comparing concordance trees to species trees as understood within the framework of the multispecies coalescent models popular in systematics today.

\section{The Relationship between Concordance Trees and Species Trees}

Now that we have formally defined concordance factors and concordance trees, we can ask what role they might play in systematics. Cranston et al. (2009), Liu et al. (2009), Leaché and Rannala (2011) and many others treat the primary concordance tree as an estimate of the species tree. By the "species tree", these authors have in mind the internodal framework described at the beginning of the paper where organisms interbreed with others inside their branch but not with those in other branches. 1 What these authors show is that as an estimate of the species tree, the concordance tree is often a good estimate, but it is not always the best estimate we can make. For example, Leaché and Rannala (2011) simulate data sets using the multispecies coalescent with a variety of tree heights and population sizes. They then use a variety of computer software programs to estimate the species tree (which is known), including using BUCKy to build a concordance tree (so actually, a greedy consensus tree), which they treat as the estimate of the species tree. While BUCKy does perform well in a variety of different circumstances, there are specific circumstances (high population sizes and short tree heights on asymmetric topologies) where BUCKy is outperformed by BEST ("Bayesian Estimation of Species Trees" as developed in Liu 2008).

It is worth pausing here to note that one might think that we have theoretical reasons for thinking that the concordance tree can't possibly be a consistent estimator of the species tree (a consistent estimator is one that will converge on the true species tree as the input data increases indefinitely). For example, it is obvious that the concordance tree can differ from the species tree if we just imagine massive amounts of lateral gene transfer. The concordance tree follows the genes, whereas species trees simply ignore this kind of genetic inheritance. But this leaves open the question of whether differences between the concordance tree and the species tree can be due entirely to factors such as incomplete lineage sorting.

Some theoretical results that initially seem relevant here include Degnan and Rosenberg (2006) who show that when the species tree has certain internal branch lengths (the "anomalous zone") then according to a standard multispecies coalescent model, the gene tree that is expected to have the highest frequency has a different topology from the species tree. Of course, by chance, the most common gene tree can always have a different topology than the species tree, but here is a case where this is expected to happen. Degnan and Rosenberg $(2006,2009)$ say that this shows that the straightforward "democratic voting" scheme for transforming gene trees into the species tree will not work. However, we must be careful here since the concordance tree uses a different kind of democratic voting procedure. It is important to distinguish fundamentally different rules that construct the concordance tree clade-by-clade (as concordance rules do) from a rule that

1. The basic framework for the multispecies coalescent models assumes the internodal picture that we just mentioned above. But numerous papers have relaxed various assumptions to try to take into account various sources of gene flow between branches (e.g., Kubatko 2009; Yu et al. 2011; Leaché et al. 2013).

๑ OPEN ACCESS - PTPBIO.ORG 
looks only at the frequencies of whole trees. For example, it is possible that the concordance tree shares no clades at all with the most frequent gene tree. So facts about the most frequent gene trees don't immediately imply anything about what the concordance tree looks like. The results of Degnan and Rosenberg (2006) leave it as an open question whether the concordance tree could differ from the species tree when we make the standard assumptions of the multispecies coalescent framework, such as that there is no selection, no lateral gene transfer, and correctly inferred gene trees. If the trees could differ in that case, then concordance analysis cannot be a statistically consistent estimator of the species tree.

It turns out that the answer is that even in this idealized framework, the concordance tree can differ from the species tree. To see this, examining some theorems from Allman et al. (2011) and Degnan et al. (2009) will prove especially valuable.

As an important preliminary, we note that in these papers cited above, the "probability of a clade" does not refer to the probability that a group actually forms a clade, but rather the expected proportion of the gene trees which actually exhibit that clade under the multispecies coalescent model that describes lineage sorting. In this setting, the "probability of a clade" is thus equal to the expected concordance factor of the group that forms that clade under this model.

Theorem 1 of Degnan et al. (2009) shows that for any number of taxa, under the multispecies coalescent model, the majority consensus tree cannot contain any clades that do not appear on the species tree. Allman et al. (2011, Theorems 4 and 5) strengthen this result and show that clades with probability $>1 / 3$ must be clades on the species tree. However, it is possible for clades on the concordance tree to have probability less than $1 / 3$, but $1 / 3$ is the lowest possible threshold where clades are guaranteed to be on the species tree. Thus we would have to examine specific cases where some clades on the concordance tree have probability less than $1 / 3$ on a case-by-case basis to see if they are also on the species tree. We have already noted that Degnan et al. (2009) show that the greedy consensus tree can differ, asymptotically, from the species tree, but in the case they describe, the problematic clade would not be on the primary concordance tree.

Nevertheless, by taking more extreme branch lengths than they discuss, it is possible to force the concordance tree for four taxa with a species tree of the form $(((a b) c) d)$ to asymptotically contain a clade (namely $(c d)$ ) that is not contained in the species tree. Allman et al. (2011) table A1 (see figure 1 below) presents a user-friendly way to display the clade probabilities under the multispecies coalescent model for every possible clade in a four taxon topology. Using this table, it can be calculated that with branch lengths of, say, $x=1$ and $y=0.01$, then the clade with the highest concordance factor is $c f(a b) \approx 0.71$ while the second highest is $c f(c d) \approx 0.29$ which barely beats out $c f(a b c) \approx 0.28$. Thus the concordance tree is $(a b)(c d)$, while the species tree is $(((a b) c) d)$.

So the concordance tree can come apart from the species tree even in the most idealized of cases. Does this mean that there is a flaw in concordance analysis? Not at all. On the concordance view, the concordance tree might not be the same as the species tree, but it can't possibly be a bad estimate of the phylogeny-it just is the phylogeny.

The right way to understand concordance views is to see them as trying to answer to the question of what a phylogeny (and a clade and a taxon) really is. Concordance analysis is not meant to simply be an inference method designed to infer the species tree. To clarify this interpretive question, Baum (2007) includes an entire section where we start by assuming we have a known population history. "Imagine that one had access to the complete population history for a group of extant populations, which is to say, for all times in the past, one knew the assignment of individuals to interbreeding populations, the descent relations of those populations, 
Figure 1: Table A1 from Allman et al. (2011,104). Here $x$ and $y$ are branch lengths given in coalescent units. Coalescent units are calculated as the ratio of the length of internodes in the species tree as measured in generations over the effective population size, as measured in individuals, of ancestral species during those internodes. Figure reprinted with permission from Elsevier.

\section{Table A1}

Probabilities of clades under three 4-taxon species trees. $X=\exp (-x), Y=\exp (-y)$.

\begin{tabular}{llll}
\hline \multirow{2}{*}{ Clade } & \multicolumn{2}{l}{ Probability under species tree } \\
\cline { 2 - 4 } & $(((a, b): x, c): y, d)$ & $((a, d): x,(b, c): y)$ & $((a, b): x,(c, d): y)$ \\
\hline$c_{1}=\mathbb{P}_{\sigma}(A B)$ & $1-\frac{2}{3} X-\frac{1}{9} X Y^{3}$ & $\frac{2}{9} X Y$ & $1-\frac{2}{3} X-\frac{1}{9} X Y$ \\
$c_{2}=\mathbb{P}_{\sigma}(A C)$ & $\frac{1}{3} X-\frac{1}{9} X Y^{3}$ & $\frac{2}{9} X Y$ & $\frac{2}{9} X Y$ \\
$c_{3}=\mathbb{P}_{\sigma}(A D)$ & $\frac{1}{6} X Y+\frac{1}{18} X Y^{3}$ & $1-\frac{2}{3} X-\frac{1}{9} X Y$ & $\frac{2}{9} X Y$ \\
$c_{4}=\mathbb{P}_{\sigma}(B C)$ & $\frac{1}{3} X-\frac{1}{9} X Y^{3}$ & $1-\frac{2}{3} Y-\frac{1}{9} X Y$ & $\frac{2}{9} X Y$ \\
$c_{5}=\mathbb{P}_{\sigma}(B D)$ & $\frac{1}{6} X Y+\frac{1}{18} X Y^{3}$ & $\frac{2}{9} X Y$ & $\frac{2}{9} X Y$ \\
$c_{6}=\mathbb{P}_{\sigma}(C D)$ & $\frac{1}{3} Y-\frac{1}{6} X Y+\frac{1}{18} X Y^{3}$ & $\frac{2}{9} X Y$ & $1-\frac{2}{3} Y-\frac{1}{9} X Y$ \\
$c_{7}=\mathbb{P}_{\sigma}(A B C)$ & $1-\frac{2}{3} Y-\frac{1}{3} X Y+\frac{1}{6} X Y^{3}$ & $\frac{1}{3} X-\frac{1}{6} X Y$ & $\frac{1}{3} Y-\frac{1}{6} X Y$ \\
$c_{8}=\mathbb{P}_{\sigma}(A B D)$ & $\frac{1}{3} Y-\frac{1}{6} X Y$ & $\frac{1}{3} Y-\frac{1}{6} X Y$ & $\frac{1}{3} Y-\frac{1}{6} X Y$ \\
$c_{9}=\mathbb{P}_{\sigma}(A C D)$ & $\frac{1}{6} X Y$ & $\frac{1}{3} Y-\frac{1}{6} X Y$ & $\frac{1}{3} X-\frac{1}{6} X Y$ \\
$c_{10}=\mathbb{P}_{\sigma}(B C D)$ & $\frac{1}{6} X Y$ & $\frac{1}{3} X-\frac{1}{6} X Y$ & $\frac{1}{3} X-\frac{1}{6} X Y$ \\
\hline
\end{tabular}

and the extent of gene flow between populations" (418). Note that this population history just is (or entails) the species tree that the multispecies coalescent views are trying to infer. Baum then wants to use the population history to try to infer the primary concordance tree. Clearly, the primary concordance tree is not meant to be an estimate of the population history. Baum (2009) and Velasco (2010) are clearer on this point, where the very meaning of "taxon", "clade" and "species" is tied to the primary concordance tree. So the primary concordance tree is meant to be an object of interest in itself and not merely (or even primarily) a useful means to infer the species tree as defined by the population history and organismal genealogies.

Usually, the primary concordance tree will have the same topology as the species tree. But it is possible that they differ. To see how this might happen, lets examine the case we constructed above. We can imagine an initial population, with a lineage $d$ splitting off and then $c$ splitting off almost immediately after that. Much later, $a$ and $b$ split, leading to the $(((a b) c) d)$ species tree. If the branch lengths are just right, we expect more genes trees to exhibit the $(c d)$ clade than the contradictory clade $(a b c)$ (which is on the species tree). It is plausible in this case to think of $c$ and $d$ as being united in a clade. Since the concordance factors of $(c d)$ and $(a b c)$ are very close in value in all such cases, it is also plausible that we should recognize neither $c d$ nor $a b c$ as a clade but rather think of the phylogeny as $(a b) c d$. This is what we would get if, for example, we required that all clades on the tree are both plurality clusters and also $R^{*}$ clusters.

Once we distinguish concordance trees from species trees, we can ask what the role is that each might play in systematics. Interestingly, many authors seem to take it as clear that the species tree is what is of primary interest to systematists and not, for example, gene trees (Ed- 
wards 2009). But Baum takes it as equally obvious that it is the gene trees that are of interest and thus concordance trees, which directly represent and summarize these gene histories, are correspondingly valuable. It seems to me (and, I suspect, to defenders of each of the above views) that both kinds of trees are of interest, even if, perhaps, for different purposes.

But when the concordance tree and the species tree come apart, which represents the phylogeny of the group? Assuming that terms like "clade" are tied to phylogeny, this is the same as asking what makes a group a clade. In the case above, $(a b c)$ is a clade on the species tree because there is a branch on the species tree leading just to that group which is the traditional definition of a clade. But why are clades important? This is related to the question of why it is important to look at phylogenetic trees at all. There are many reasons to do so-see, for example, Baum and Smith (2012) for a comprehensive study. As an illustrative example, think about character evolution. If we find a trait in humans and gorillas but not chimps and we know that humans and chimps are part of a clade that does not include gorillas, then we will infer that either the trait evolved twice separately in the human and gorilla lineages or the trait was lost in the chimp lineage. This is because we assume that traits follow the history of the species and the lineage splits. However, if they come apart, this can be a mistaken inference. As a matter of fact, a study of great apes found that among 11,945 gene trees with high posterior probability, $76.6 \%$ supported the ((human,chimp),gorilla) relationship, whereas $11.5 \%$ and $11.4 \%$ supported the ((chimp,gorilla), human) and ((human,gorilla),chimp) relationships, respectively (Ebersberger et al. 2007). In this case, the dominant genetic history represented by the primary concordance tree follows what we believe to be the species tree so this is the best we can do if we want to infer what the history of the trait is. However, imagine that the concordance tree came apart from the species tree. If true species history was ((human,chimp),gorilla) but the majority of the genes followed the ((human,gorilla),chimp) tree, then we should not infer that a trait found only in humans and gorillas evolved twice. In that case, using the species tree instead of the concordance tree for studying character evolution would be positively misleading. It is important to know which groups are clades because we assume that clades have a unique shared history. But if we want to know about their shared genetic history we want to know which groups are clades on the concordance tree and not necessarily on the species tree. While it is true that in many cases, genetic discordance means that no group has a completely shared genetic history, concordance trees represent the best that we can do in representing the dominant history.

It is important to point out here that concordance trees are no panacea here. For example, Hahn and Nakhleh (2016) look at the case of the evolution of echolocation in bats. Bat phylogeny has been very controversial, but a number of studies indicate that the microbats (those that echolocate) are in fact a paraphyletic group which would seem to indicate that echolocation either evolved twice or was lost in another group of bats (in this case, the non-echolocating Old World fruit bats). However, Hahn and Nakhleh point out that there is a high degree of genetic discordance between gene trees in this phylogeny such that $22.3 \%$ of the sampled genes actually show the microbats forming a clade and as such it is extremely plausible that key genes involved in echolocation in fact evolved just once and simply follow this minority gene tree. They argue that the search for a single, resolved species tree which then gets used exclusively for studies of the group is seriously problematic. In this they are entirely correct. If we were forced to use just one tree, we should use the primary concordance tree- the tree that gets the most gene history right. However, the better solution is to just recognize that there is no reason to use just one resolved tree; rather, we should recognize that "phylogenetic incongruence [is] a signal, rather than a problem" (Nakhleh 2013). In fact, the problem of reticulation and discordant gene trees is exactly what led to the study of concordance factors in the first place. 
When making inferences about evolutionary history, it is essential that we recognize that different genes have different histories. But sometimes it is useful to summarize this history in a single tree. If we do summarize genetic history using just a single tree, then that tree should represent the dominant history-the tree that gets more genetic history right than any other tree. This is just what the primary concordance tree represents. One reason to have a single tree is that it can serve as the basis for taxonomy. While different genes have different histories, there are good reasons to have a single, all purpose classification system and there are good reasons to base this taxonomic system on phylogeny (Hennig 1966; Baum and Smith 2013). This phylogeny should track the actual flow of genetic information and thus should be based on the primary concordance tree. As Baum (2007) puts it, "While some clades on a primary concordance tree will be too transient or local to warrant formal recognition, it is hard to see why we would wish to name any clade that is not on the true primary concordance tree of life" (425). But this is just what happens if we define species or phylogenies more generally according to species trees. So while there is also some reason to think of branches on the species tree but not the concordance tree as clades, I do not think there is any particular reason to think that the species tree must be the tree that determines the meaning of "phylogeny" in these case and it is certainly not the case that the concordance tree is of no independent interest.

I also want to stress that the case constructed above was in some sense a best-case scenario for the use of the species tree-one where the difference between the species tree and the concordance tree was minimal and entirely due to incomplete lineage sorting. With random fluctuations due to a finite amount of data, some gene histories being influenced by natural selection, or even instances of non-random mating, the distribution of gene trees will differ from what is expected to hold in the standard multispecies coalescent framework. The more expectations differ from actuality, the more important tracking the actual gene histories seems to be. In cases such as hybridization across lineages, it is not even clear what the species tree is or how to define it. The concordance tree is at least defined and forms a tree in all of these cases and if the gene flow is significant enough, it makes sense to us to think that it actually changes the phylogeny. While we do not think these considerations give us any definitive reason to favor the examination of concordance trees instead of building species trees, we do think there are serious reasons to investigate concordance trees apart from their use as estimators of species trees.

\section{Conclusion}

We have seen that the idea of a tree of life representing phylogenetic history can be realized in a number of different ways. On a concordance view, phylogenetic history is represented by the dominant phylogenetic histories of the genes. The primary concordance tree is a useful summary of phylogenetic history and serves quite well for many traditional phylogenetic purposes. But it is important to recognize how this tree differs from other ways of representing genealogical history, whether it be tracking population history through time or reducing the network of organismal genealogical connections to a hierarchy of clusters. The primary concordance tree is not merely valuable as an estimate of the species tree, as some authors have assumed, but an important object of study in its own right. And when it does come apart from internodal species trees based on organismal networks or from species trees built by assuming the framework of the multispecies coalescent, there are strong reasons to think that the primary concordance tree fulfills the role of phylogeny better than any of these alternatives. 


\section{Acknowledgments}

The deepest thanks goes to Mike Steel who was a co-author on an ancestral version of this paper. Parts of that paper were published as Steel and Velasco (2014). In addition, I would like to thank Cecile Ané, David Baum, James Degnan, Kenny Easwaran, Matt Haber, and anonymous referees who read and commented on this paper or on a relevant ancestral version. Thanks also go to audience members at the "Species in the Age of Discordance" Conference (23-25 March 2017) at the University of Utah. Thanks also to support from the National Science Foundation (award \#1557117, "Evolution and the Levels of Lineage").

\section{Literature cited}

Alexander, S. 2012. “Infinitary Species: An Idealized Mathematical Species Concept.” arXiv:1201.2869. http://cds.cern.ch/record/1416250.

Allman, E. S., J. H. Degnan, and J. A. Rhodes. 2011. "Determining Species Tree Topologies from Clade Probabilities under the Coalescent.” J. Theor. Biol. 289:96-106. doi:10.1016/j.jtbi.2011.08. 006.

Ané, C. 2010. "Reconstructing Concordance Trees and Testing the Coalescent Model from GenomeWide Data Sets.” Chap. 3 in Estimating Species Trees: Practical and Theoretical Aspects, edited by L. L. Knowles and L. S. Kubatko. Wiley-Blackwell.

Ané, C. 2019. BUCKy: A Program for Bayesian Concordance Analysis. Accessed May. http://pages.stat. wisc.edu/ ane/bucky/index.html.

Ané, C., B. Larget, D. A. Baum, S. D. Smith, and A. Rokas. 2007. "Bayesian Estimation of Concordance among Gene Trees.” Mol. Biol. Evol. 24 (2): 412-426. doi:10.1093/molbev/msl170.

Barrett, M., M. J. Donoghue, and E. Sober. 1991. “Against Consensus.” Syst. Biol. 40 (4): 486-493.

Baum, D. A. 2007. "Concordance Trees, Concordance Factors, and the Exploration of Reticulate Genealogy.” Taxon 56:417-426. doi:10.1002/tax.562013.

Baum, D. A. 2009. “Species as Ranked Taxa.” Syst. Biol. 58 (1): 74-86. doi:10.1093/sysbio/syp011.

Baum, D. A., and K. L. Shaw. 1995. "Genealogical Perspectives on the Species Problem." In Experimental and Molecular Approaches to Plant Biosystematics, edited by P. C. Hoch and A. G. Stephenson. St. Louis, MO: Missouri Botanical Garden Press.

Bininda-Emonds, O. R. P. 2004. Phylogenetic Supertrees. Kluwer.

Bryant, D. 2003. “A Classification of Consensus Methods for Phylogenies.” In Bioconsensus, edited by M. Janowitz, F.-J. Lapointe, F. R. Mcmorris, B. Mirkin, and F. S. Roberts, 163-84. DIMACS Series in Discrete Mathematics and Theoretical Computer Science 61.

Bui-Xuan, B.-M., M. Habib, and M. Rao. 2012. "Tree-Representation of Set Families and Applications to Combinatorial Decompositions." Eur. J. Combin. 33 (5): 688-711. doi:10.1016/j. ejc. 2011.09.032.

Cranston, K. A., B. Hurwitz, D. Ware, L. Stein, and R. A. Wing. 2009. "Species Trees from Highly Incongruent Gene Trees in Rice.” Syst. Biol. 58 (5): 489-500. doi:10.1093/sysbio/syp054.

De Queiroz, K., and M. Donoghue. 1990. "Phylogenetic Systematics or Nelson's Version of Cladistics?" Cladistics 6:61-75. doi:10.1111/j.1096-0031.1990.tb00525.x.

Degnan, J. H., M. Degiorgio, D. Bryant, and N. A. Rosenberg. 2009. "Properties of Consensus Methods for Inferring Species Trees From Gene Trees.” Syst. Biol. 58:35-54.

○ OPEN ACCESS - PTPBIO.ORG 
Degnan, J. H., and N. A. Rosenberg. 2006. "Discordance of Species Trees with Their Most Likely Gene Trees.” PLoS Genet. 2:e68. doi:10.1371/journal.pgen.0020068.

Degnan, J. H., and N. A. Rosenberg. 2009. "Gene Tree Discordance, Phylogenetic Inference and the Multispecies Coalescent.” Trends Ecol. Evol. 24 (6): 332-340. doi:10.1016/j.tree.2009.01.009.

Dong, J., D. Fernándes-Baca, F. R. Mcmorris, and R. C. Powers. 2010. "Majority-Rule (+) Consensus Trees." Math. Biosci. 228:10-15. doi:10.1016/j.mbs.2010.08.002.

Donnelly, K. P. 1983. "The Probability That Related Individuals Share Some Section of Genome Identical by Descent.” Theor. Popul. Biol. 23 (1): 34-63. doi:10.1016/0040-5809(83)90004-7.

Dress, A. W. M., V. Moulton, M. Steel, and T. Wu. 2010. "Species, Clusters and the 'Tree of Life': A Graph-Theoretic Perspective.” J. Theor. Biol. 265 (4): 535-542. doi:10.1016/j.jtbi.2010.05.031.

Ebersberger, I., P. Galgoczy, S. Taudien, S. Taenzer, M. Platzer, and A. Von Haeseler. 2007. "Mapping Human Genetic Ancestry.” Molecular Biology and Evolution 24 (10): 2266-2276. doi:10.1093/ molbev/msm156.

Edwards, S. V. 2009. "Is a New and General Theory of Systematics Emerging?" Evolution 63 (1). doi:1 0.1111/j.1558-5646.2008.00549.x.

Goloboff, P. A., J. S. Farris, and K. C. Nixon. 2008. “TNT, a Free Program for Phylogenetic Analysis.” Cladistics 24 (5): 774-786. doi:10.1111/j.1096-0031.2008.00217.x.

Hahn, M. W., and L. Nakhleh. 2016. "Irrational Exuberance for Resolved Species Trees." Evolution 70 (1): 7-17. doi:10.1111/evo.12832.

Hennig, W. 1966. Phylogenetic Systematics. Urbana: University of Illinois Press.

Hudson, R. R., and J. A. Coyne. 2002. "Mathematical Consequences of the Genealogical Species Concept.” Evolution 56 (8): 1557-1565. doi:10.1554/0014-3820(2002)056[1557:mcotgs]2.0.co; 2 .

Kornet, D. J. 1993. "Permanent Splits as Speciation Events: A Formal Reconstruction of the Internodal Species Concept.” J. Theor. Biol. 164:407-435. doi:10.1006/jtbi.1993.1164.

Kornet, D. J., J. A. J. Metz, and H. A. J. M. Schellinx. 1995. "Internodons as Equivalence Classes in Genealogical Networks: Building-Blocks for a Rigorous Species Concept.” J. Math. Biol. 34:110122.

Kubatko, L. S. 2009. "Identifying Hybridization Events in the Presence of Coalescence via Model Selection.” Systematic Biology 58 (5): 478-488. doi:10.1093/sysbio/syp055.

Larget, B. R., S. K. Kotha, C. N. Dewey, and C. Ané. 2010. "BUCKy: Gene Tree/Species Tree Reconciliation with Bayesian Concordance Analysis.” Bioinformatics 26 (22): 2910-2911. doi:10. 1093/bioinformatics/btq539.

Leaché, A. D., and B. Rannala. 2011. "The Accuracy of Species Tree Estimation Under Simulation: A Comparison of Methods.” Systematic Biology 60:126-137. doi:10.1093/sysbio/syq073.

Leaché, A. D., R. B. Harris, B. Rannala, and Z. Yang. 2013. "The Influence of Gene Flow on Species Tree Estimation: A Simulation Study.” Systematic Biology 63 (1): 17-30. doi:10.1093/sysbio/ syt049.

Liu, L. 2008. “BEST: Bayesian Estimation of Species Trees Under the Coalescent Model.” Bioinformatics 24:2542-2543. 
Liu, L., L. Yu, L. Kubatko, D. K. Pearl, and S. V. Edwards. 2009. "Coalescent Methods for Estimating Phylogenetic Trees.” Mol. Phylogenet. Evol. 53 (1): 320-328. doi:10.1016/j.ympev.2009.05. 033.

Maddison, W. P. 1995. "Phylogenetic Histories within and among Species." In Experimental and Molecular Approaches to Plant Biosystematics, edited by P. C. Hoch and A. G. Stephenson. St. Louis: Missouri Botanical Garden.

Maddison, W. P. 1997. “Gene Trees in Species Trees.” Syst. Biol. 46 (3): 523-536. doi:10.2307/241369 4.

Nakhleh, L. 2013. "Computational Approaches to Species Phylogeny Inference and Gene Tree Reconciliation." Trends in Ecology and Evolution 28 (12): 719-728. doi:10.1016/j.tree.2013.09.004.

Puigbò, P., Y. I. Wolf, and E. V. Koonin. 2009. "Search for a 'Tree of Life' in the Thicket of the Phylogenetic Forest.” Journal of Biology 8 (59). doi:10.1186/jbiol159.

Ridley, M. 1989. “The Cladistic Solution to the Species Problem.” Biology and Philosophy 4 (1): 1-16.

Roch, S., and S. Snir. 2013. "Recovering the Tree-Like Trend of Evolution Despite Extensive Lateral Genetic Transfer: A Probabilistic Analysis.” J. Comput. Biol. 20 (2): 93-112.

Steel, M. 2006. "Tools to Construct and Study Big Trees: A Mathematical Perspective." Chap. 7 in $R e-$ constructing the Tree of Life: Taxonomy and Systematics of Species Rich Taxa, edited by T. Hodkinson and J. Parnell. Systematics Association Special Volumes. CRC Press.

Steel, M. 2007. "Tools to Construct and Study Big Trees: A Mathematical Perspective." Systematics Association Special Volume 72:97.

Steel, M., S. Linz, D. H. Huson, and M. J. Sanderson. 2013. "Identifying a Species Tree Subject to Random Lateral Gene Transfer.” J. Theor. Biol. 322:81-93. doi:10.1016/j.jtbi.2013.01.009.

Steel, M., and J. D. Velasco. 2014. "Axiomatic Opportunities and Obstacles for Inferring a Species Tree From Gene Trees.” Systematic Biology 63 (5): 772-778. doi:10.1093/sysbio/syu043.

Tsagkogeorga, G., J. Parker, E. Stupka, J. A. Cotton, and S. J. Rossiter. 2013. "Phylogenomic Analyses Elucidate the Evolutionary Relationships of Bats." Current Biology 23 (22): 2262-2267. doi:10. 1016/j.cub.2013.09.014.

Velasco, J. D. 2009. "When Monophyly Is Not Enough: Exclusivity as the Key to Defining a Phylogenetic Species Concept.” Biol. Philos. 24 (4): 473-486. doi:10.1007/s10539-009-9151-4.

Velasco, J. D. 2010. “Species, Genes, and the Tree of Life.” Brit. J. Philos. Sci. 61 (3): 599-619. doi:10. 1093/bjps/axp051.

Velasco, J. D. 2013. "Phylogeny as Population History." Philosophy E Theory in Biology 5 (2). doi:10. 3998/ptb.6959004.0005.002.

Williams, D., G. P. Fournier, P. Lapierre, K. S. Swithers, A. G. Green, C. P. Andam, and J. P. Gogarten. 2011. “A Rooted Net of Life." Biol. Direct 6:45. doi:10.1186/1745-6150-6-45.

Yu, Y., C. Than, J. H. Degnan, and L. Nakhleh. 2011. "Coalescent Histories on Phylogenetic Networks and Detection of Hybridization Despite Incomplete Lineage Sorting." Systematic Biology 60 (2): 138-149. doi:10.1093/sysbio/syq084.

(c) 2019 Author(s) This is an open-access article distributed under the terms of the Creative Commons Attribution 4.0 International license, which permits anyone to download, copy, distribute, display, or adapt the text without asking for permission, provided that the creator(s) are given full credit.

ISSN 2475-3025

๑ OPEN ACCESS - PTPBIO.ORG 